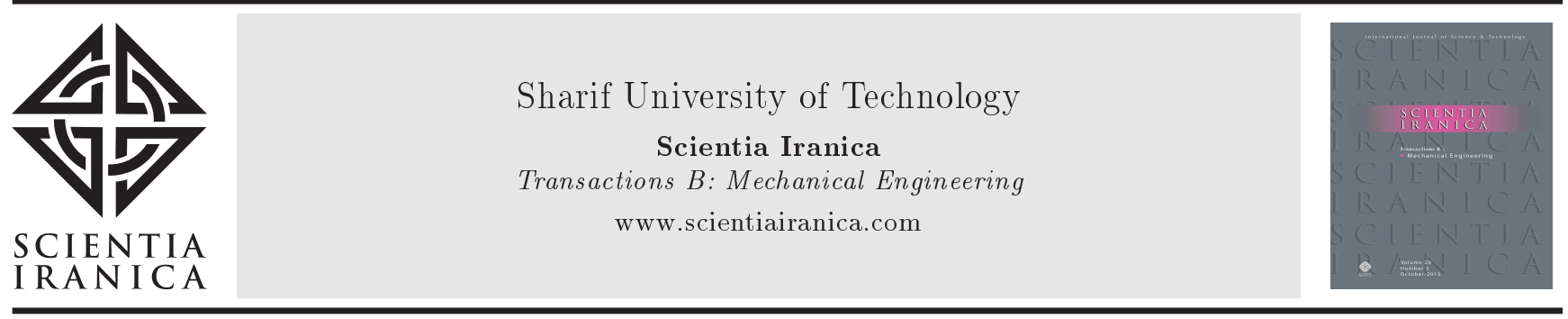

\title{
Modeling the size dependent pull-in instability of cantilever nano-switch immersed in ionic liquid electrolytes using strain gradient theory
}

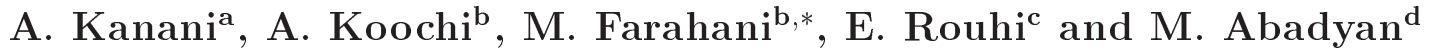 \\ a. Ionizing and Non-Ionizing Radiation Protection Research Center, Paramedical Sciences School, Shiraz University of Medical \\ Sciences, Shiraz, Iran. \\ b. Department of Aerospace Engineering, Sharif University of Technology, Tehran, P.O. Box 11365-11155, Iran. \\ c. Young Researchers and Elite Club, Mashhad Branch, Islamic Azad University, Mashhad, Iran. \\ d. Shahrekord Branch, Islamic Azad University, Shahrekord, Iran.
}

Received 14 March 2014; received in revised form 18 October 2014; accepted 7 March 2016
KEYWORDS
Cantilever NEMS;
Ionic liquid
electrolyte;
Pull-in instability;
Strain gradient
theory;
Dispersion forces.

\begin{abstract}
It is well recognized that size-effect often plays a significant role in the mechanical performance of nano-structures. Herein, strain gradient continuum elasticity is employed to investigate the size dependent pull-in instability of the cantilever nanoactuators immersed in ionic liquid electrolyte. The presence of dispersion forces, i.e. Casimir and van der Waals field, is considered in the theoretical model as well as the double-layer electrochemical attraction. To solve the non-linear constitutive equation of the system, two approaches, i.e. the Rayleigh Ritz Method (RRM) and the numerical solution method, are employed. Impact of the size dependency and dispersion forces on the instability characteristics are discussed as well as the effect of ion concentration in liquid.

(C) 2016 Sharif University of Technology. All rights reserved.
\end{abstract}

\section{Introduction}

Micro/nano-electromechanical systems (MEMS/NEMS) are increasingly used in various engineering and science branches, i.e. mechanics, chemistry, optics, biology, electronics, etc. These ultra-small systems are employed to develop nano-devices such as sensors, actuators, accelerometer, tweezers, switches, etc. [1]. A typical MEMS/NEMS is constructed from movable conductive component(s), which can be excited via applying electrical Coulomb force field. The electrical stimulation can result in deflection, vibration, or actuation of the movable component(s), depending

\footnotetext{
*. Corresponding author. Tel.: +9821 66022731;

Fax: +982166022731

E-mail addresses: en.a.kanani@gmail.com (A. Kanani);

A.koochi@Gmail.com (A.Koochi); mfarahani@sharif.edu,

(M. Farahani); eh.roohi@gmail.com (E. Rouhi);

abadyan@yahoo.com (M. Abadyan)
}

on the system design. Generally, when the applied voltage exceeds its critical value, the pull-in instability occurs and the MEMS/NEMS suddenly fails. Prediction and simulation of the pull-in instability of MEMS/NEMS are very crucial for reliable design and fabrication of nano-devices; hence much research is dedicated to study the pull-in behavior of the ultrasmall systems [2,3]. It should be noted that for micro- and nano-beam, the electrical field is not exactly homogenous and therefore the effects of fringing fields on the electrostatic force are not negligible because of the nonzero thickness and finite width of beams [4].

With recent developments in biological, chemical, and electronic sciences, NEMS/MEMS has found many applications in liquid media. Some of the promising applications of MEMS/NEMS in bio-fluid are developing sensors and manipulators for cellular handling, bio-component characterization, device motion, DNA manipulation, bio-mimetic cilia, drug 
delivery, etc. [5-8]. Besides biology, nano-devices such as nano-actuators, nano-switches, nano-probes, nano-tweezers, micro-valves, micro-gears, etc. are employed as precise instruments operated in ionic liquid media [9]. Moreover, usage of in-liquid immersed MEMS/NEMS has great potentials in many electrochemical and electronic applications including super-capacitors, fuel cells, batteries, filters, microdensitometers, micro/nano-pumps, active microfiuidic devices, and atomic force microscopy [9-11]. In this regard, the previous researchers have investigated the mechanical behavior of MEMS/NEMS in liquid environment $[7,9,12]$. Oh et al. [7] have fabricated and characterized the oscillating bio-mimetic microfluidic device that mimics biological cilia for manipulation of microfluidics. Abdelhamid Maali et al. attempted to measure the influence of the fluid motion on the hydrodynamics and oscillating behavior of an atomic force microscopy cantilevers immersed in viscous fluids [13]. The stability behavior and pull-in performance of various types of electrostatic parallel-plate actuators in liquid solutions were studied by Rollier et al. [5]. They have claimed that pull-in instability can be shifted far beyond one-third of the initial gap and can even be suppressed in liquid. Only few works have focused on the pull-in behavior of liquid-immersed NEMS in ionic environment where the electrochemical field is characterized by double-layer interaction [9,12]. A simple lumped model for the calculation of pull-in voltage of electrostatic actuators in ionic liquid electrolytes was presented by Boyd and Kim [9]. They incorporated the effect of doublelayer electrochemical force in the pull-in model by solving the linearized Poisson-Boltzmann equation. In another work, Boyd and Lee [14] have modified their model with a distributed parameter model in order to achieve more accurate results. The electromechanical behavior and frequency response of an inter-digitated silicon comb-drive actuator in various ionic liquids were investigated by Sounart et al. [12]. They presented a theoretical model that predicted the characteristic actuation frequency of the system. Noghrehabadi et al. [15] theoretically investigated the static pull-in instability of nano-beams in a liquid electrolyte using a distributed force model. They applied Adomian method to solve the governing non-linear equation of the system. The influences of hydration force and elastic strain energy on stability of solid films in thin solid-on-liquid structure were investigated in [16].

It is well established that the electromagnetic quantum fluctuations, i.e. dispersion forces, can significantly affect the electromechanical performance of nano-structures. Hence, incorporating the effect of dispersion forces is crucial to accurate simulation of nano-structures in liquid media. The dispersion forces between interacting bodies are generally explained as
Casimir or van der Waals (vdW) attractions depending on the distance between the bodies. If the separation between the interacting bodies is sufficiently large (typically of the order of hundreds of nanometers), the retardation effect is significant and the fluctuation force is modeled as Casimir force. Many researchers have experimentally [17,18] and theoretically [19-29] investigated the effect of Casimir force on the performance of ultra-small systems. Lamoreaux presented a comprehensive review of the theory of Casimir force and its corrections for real material and finite temperature [30]. A comprehensive investigation of the Casimir force between interacting bodies can be found in [27]. However, if the separation between the interacting bodies is typically less than few nanometers, the retardation effect is negligible and the nano-scale interaction is modeled as the $\mathrm{vdW}$ attraction regime. The pull-in instability of NEMS in the presence of vdW force has been studied by previous researchers using various approaches [31-36].

To investigate the electromechanical performance of MEMS/NEMS in liquid, continuum mechanic models can be applied [14]. Since the elastic characteristics of materials in nano-scale are size-dependent $[37,38]$, the applied models should be able to consider this sizedependency in constitutive equations. Lam et al. [39] observed that the normalized bending rigidity of the epoxy polymeric micro-beams becomes 2.4 times larger when the thickness of the beam decreases from $115 \mathrm{~mm}$ to $20 \mathrm{~mm}$. The bending of polypropylene micro-sized cantilever beams has been experimentally investigated by McFarland and Colton [40]. They observed that the stiffness of micro-sized cantilevers was at least four times larger than the value which the classical theory of elasticity anticipated. By applying nonclassic continuum theories, the size dependent behavior of nano-structures is attributed to material lengthscale parameters. A length-scale parameter might be considered as a mathematical parameter that scales the strain gradients in the constitutive model so as to balance the dimensions of strains $(\varepsilon)$ and strain gradients $(d \varepsilon / d x)[41]$. As the characteristic length of the deformation field becomes significantly larger than the material length-scale parameter, strain gradient effects become negligible, because the strain terms are much larger than their scaled gradient terms [41]. Some experimental measurements evaluate the material lengthscale parameters of single crystal and polycrystalline copper to be 12 and $5.84 \mu \mathrm{m}$, respectively [42,43]. Also, the size-dependent behavior has been detected in some kinds of polymers [44]. For hardness measurement of gold bulk, it is found that the plastic length-scale parameter (for indentation test and hardness behavior) of $\mathrm{Au}$ increases from $470 \mathrm{~nm}$ to $1.05 \mu \mathrm{m}$ with increasing the $\mathrm{Au}$ film thickness from $500 \mathrm{~nm}$ to $2 \mu \mathrm{m}$ [45]. Based on test results gathered via microhardness test, 
the plastic length-scale parameters for metals such as $\mathrm{Cu}$ and $\mathrm{Ag}$ Brass were determined in the range about $0.2-20 \mu \mathrm{m}$ depending on the crystallity [46]. Using microbend testing method, the plastic intrinsic material length-scales of $4 \mu \mathrm{m}$ for copper and $5 \mu \mathrm{m}$ for nickel were determined [41]. All these experiments imply that when the characteristic size (thickness, diameter, etc.) of a micro/nano element is in the order of its intrinsic length scales (typically sub-micron), the material elastic constants highly depend on the element dimensions. Molecular dynamic simulations could also be used to compute the material length-scale parameters of materials [47].

This phenomenon cannot be conducted by using classical continuum mechanics. However, by applying non-classic continuum theories, the size dependent behavior of nano-structures is attributed to material length-scale parameters. In this regard, the non-classical theories such as non-local elasticity [48], couple stress theory [49,50], strain gradient theory [39], modified couple-stress theory [51], etc. have been developed to consider the size-effect in theoretical continuum models. While some simple size-dependent models based on modified couple-stress theory have been applied to analyze the pull-in instability of MEMS/NEMS [52-57], only rare works have utilized strain gradient theory for analyzing this phenomenon [58-63]. However, none of the abovementioned works has investigated the stability behavior of MEMS/NEMS in liquid electrolyte media.

This paper is devoted to analyze the pull-in behavior of a cantilever nano-switch immersed in liquid electrolytes in the presence of small-scale effects, i.e. the dispersion forces (Casimir and van der Waals), as well as the size-dependency of characteristics (sizeeffect). The strain gradient theory in conjunction with Euler-Bernoulli beam model is used to derive the non-linear equilibrium equation of the system. Two different methods, i.e. Rayleigh-Ritz and numerical solution methods, have been applied to solve the governing equation of the system. These two solution approaches are compared with each other as well as those presented in literature.

\section{Theoretical model}

\subsection{Fundamentals of strain gradient theory}

The mechanical behavior of ultra-small structures in micro/nano-scales significantly depends on the dimensions of the structure. In spite of the classic continuum mechanics, the strain gradient theory introduces three length-scale parameters that can model the sizedependent behavior of the ultra-small systems.

Regarding the strain gradient theory, modified and suggested by Lam et al. [39], $\bar{U}$, the stored strain energy density in the linear elastic isotropic material, is written as the followings:

$$
\bar{U}=\frac{1}{2}\left(\sigma_{i j} \varepsilon_{i j}+p_{i} \gamma_{i}+\tau_{i j k}^{(1)} \eta_{i j k}^{(1)}+m_{i j}^{s} \chi_{i j}^{s}\right),
$$

in which:

$$
\begin{aligned}
\varepsilon_{i j}= & \frac{1}{2}\left(u_{i, j}+u_{j, i}\right), \\
\gamma_{i}= & \varepsilon_{m m, i}, \\
\eta_{i j k}^{(1)}= & \frac{1}{3}\left(\varepsilon_{j k, i}+\varepsilon_{k i, j}+\varepsilon_{i j, k}\right) \\
& -\frac{1}{15} \delta_{i j}\left(\varepsilon_{m m, k}+2 \varepsilon_{m k, m}\right) \\
& -\frac{1}{15}\left[\delta_{j k}\left(\varepsilon_{m m, i}+2 \varepsilon_{m i, m}\right)\right. \\
& \left.+\delta_{k i}\left(\varepsilon_{m m, j}+2 \varepsilon_{m j, m}\right)\right], \\
\chi_{i j}^{s}= & \frac{1}{2} e_{j k l} u_{l, k i} .
\end{aligned}
$$

In the above equations, $u_{i}, \gamma_{i}, \eta_{i j k}^{(1)}, \chi_{i j}^{s}, \delta_{i j}$, and $e_{i j k}$ indicate components of displacement vector, dilatation gradient vector, deviatoric stretch gradient tensor, symmetric rotation gradient tensor, Kronocker delta, and permutation symbol, respectively. Also, $\sigma_{i j}$, $p_{i}, \tau_{i j k}^{(1)}$, and $m_{i j}^{s}$ are components of Cauchy's stress and high order stress tensors, respectively, that are identified as [39]:

$$
\begin{aligned}
& \sigma_{i j}=2 \mu\left(\varepsilon_{i j}+\frac{\nu}{1-2 \nu} \varepsilon_{m m} \delta_{i j}\right), \\
& p_{i}=2 \mu l_{0}^{2} \gamma_{i}, \\
& \tau_{i j k}^{(1)}=2 \mu l_{1}^{2} \eta_{i j k}^{(1)}, \\
& m_{i j}^{s}=2 \mu l_{2}^{2} \chi_{i j}^{s} .
\end{aligned}
$$

In the above equations, $\nu$ and $\mu$ are Poisson's ratio and shear modulus, respectively. Also, $l_{0}, l_{1}$, and $l_{2}$ are additional material length-scale parameters, which are dependent on dilatation gradient vector, deviatoric stretch gradient tensor, and symmetric rotation gradient tensor.

\subsection{Non-linear constitutive equation}

Figure 1 shows the schematic representation of beamtype nano-cantilever which is constructed from a conductive electrode suspended over another fixed one (grounded electrode). Herein, the cantilever electrode with a length of $L$, width of $b$, and thickness of $h$ is considered. Applying voltage difference between the electrodes causes deflection of the upper one toward the grounded one. 


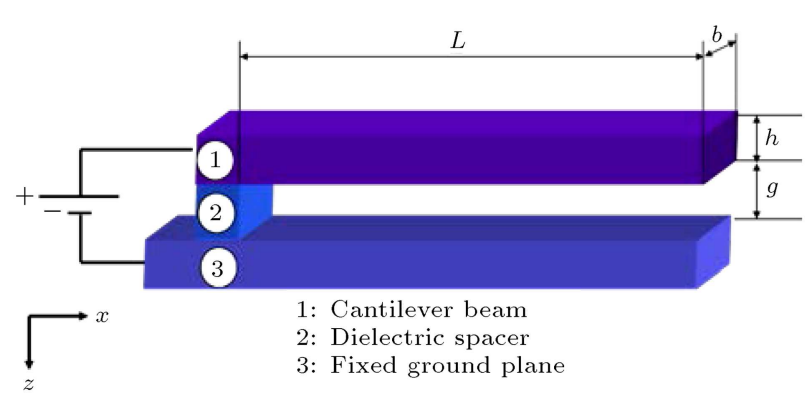

Figure 1. Schematic representation of cantilever nano-switch.

\subsubsection{Strain energy}

In this work, the displacement field of Euler-Bernoulli beam theory is applied for modeling the elastic behavior of the cantilever nano-structure. Based on this theory, the displacement field can be written as the following:

$$
u_{1}=-Z \frac{\partial W}{\partial X}, \quad u_{2}=0, \quad u_{3}=W(X) .
$$

The variable $W$ indicates beam displacement in direction of $Z$ axis. Substituting the linear displacement field of Eq. (10) in Eqs. (2)-(9), the nonzero component is obtained as the following relations:

$$
\begin{aligned}
& \varepsilon_{11}=-z \frac{\partial^{2} w(x)}{\partial X^{2}}, \\
& \sigma_{11}=-E z \frac{\partial^{2} w(x)}{\partial X^{2}}, \\
& \gamma_{1}=-z \frac{\partial^{3} w(x)}{\partial X^{3}}, \quad \gamma_{3}=-\frac{\partial^{2} w(x)}{\partial X^{2}}, \\
& p_{1}=-2 \mu l_{0}^{2} z \frac{\partial^{3} w(x)}{\partial X^{3}}, \quad p_{3}=-2 \mu l_{0}^{2} \frac{\partial^{2} w(x)}{\partial X^{2}}, \\
& \chi_{12}^{s}=\chi_{21}^{s}=-\frac{1}{2} \frac{\partial^{2} w(X)}{\partial X^{2}}, \\
& m_{12}^{s}=m_{21}^{s}=-\mu l_{2}^{2} \frac{\partial^{2} w(X)}{\partial X^{2}}, \\
& \eta_{111}^{(1)}=-\frac{2}{5} Z \frac{\partial^{3} w}{\partial X^{3}}, \\
& \eta_{113}^{(1)}=\eta_{131}^{(1)}=\eta_{311}^{(1)}=-\frac{4}{15} \frac{\partial^{2} w}{\partial X^{2}}, \\
& \eta_{122}^{(1)}=\eta_{212}^{(1)}=\eta_{221}^{(1)}=\eta_{133}^{(1)}=\eta_{313}^{(1)}=\eta_{331}^{(1)}=\frac{1}{5} Z \frac{\partial^{3} w}{\partial X^{3}}, \\
& \eta_{223}^{(1)}=\eta_{232}^{(1)}=\eta_{322}^{(1)}=\frac{1}{15} \frac{\partial^{2} w}{\partial X^{2}}, \\
& \eta_{333}^{(1)}=\frac{1}{5} \frac{\partial^{2} w}{\partial X^{2}},
\end{aligned}
$$

$$
\begin{aligned}
& \tau_{111}^{(1)}=-\frac{4}{5} \mu l_{1}^{2} Z \frac{\partial^{3} w}{\partial X^{3}} \\
& \tau_{113}^{(1)}=\tau_{131}^{(1)}=\tau_{311}^{(1)}=-\frac{8}{15} \mu l_{1}^{2} \frac{\partial^{2} w}{\partial X^{2}}, \\
& \tau_{122}^{(1)}=\tau_{212}^{(1)}=\tau_{221}^{(1)}=\tau_{133}^{(1)}=\tau_{313}^{(1)}=\tau_{331}^{(1)}=\frac{2}{5} \mu l_{1}^{2} Z \frac{\partial^{3} w}{\partial X^{3}}, \\
& \tau_{223}^{(1)}=\tau_{232}^{(1)}=\tau_{322}^{(1)}=\frac{2}{15} \mu l_{1}^{2} \frac{\partial^{2} w}{\partial X^{2}}, \\
& \tau_{333}^{(1)}=\frac{2}{5} \mu l_{1}^{2} \frac{\partial^{2} w}{\partial X^{2}} .
\end{aligned}
$$

Substituting Relations (11) after some elaboration by Eq. (1) and integrating them into the beam volume, the bending strain energy is obtained as the following:

$$
\begin{aligned}
U= & \int_{V} \bar{U} d V=\frac{1}{2} \int_{0}^{L} \int_{A}\left\{E(Z) Z^{2}\left(\frac{d^{2} W}{d X^{2}}\right)^{2}\right. \\
& +2 \mu l_{0}^{2} Z^{2}\left(\frac{d^{3} W}{d X^{3}}\right)^{2}+2 \mu l_{0}^{2} Z^{2}\left(\frac{d^{2} W}{d X^{2}}\right)^{2} \\
& +2 \mu l_{2}^{2} Z^{2}\left(\frac{d^{2} W}{d X^{2}}\right)^{2}+\frac{4}{25} \mu l_{1}^{2} Z^{2}\left(\frac{d^{3} W}{d X^{3}}\right)^{2} \\
& +\frac{48}{225} \mu l_{1}^{2}\left(\frac{d^{2} W}{d X^{2}}\right)^{2}+\frac{6}{25} \mu l_{1}^{2} Z^{2}\left(\frac{d^{3} W}{d X^{3}}\right)^{2} \\
& \left.+\frac{1}{75} \mu l_{1}^{2}\left(\frac{d^{2} W}{d X^{2}}\right)^{2}+\frac{1}{25} \mu l_{1}^{2}\left(\frac{d^{2} W}{d X^{2}}\right)^{2}\right\} d A d X \\
= & \frac{1}{2} \int_{0}^{L}\left[\left(E I+2 \mu A l_{0}^{2}+\frac{8}{15} \mu A l_{1}^{2}+\mu A l_{2}^{2}\right)\left(\frac{d^{2} W}{d X^{2}}\right)^{2}\right. \\
& \left.+\bar{A}\left(2 \mu l_{0}^{2}+\frac{4}{5} \mu l_{1}^{2}\right)\left(\frac{d^{3} W}{d X^{3}}\right)^{2}\right] d X .
\end{aligned}
$$

In the above equation, $I$ is the second cross section moment around $Y$ axis and $\bar{A}$ is the cross section area.

It should be noted that axial stretching and force resultants are not considered in deriving the governing equation of the structure due to the free end of the cantilever beam. However, in the case of doubly clamped structures, the deflection of beam induces axial stretching. Therefore, for doubly clamped structures, this axial stretching should be taken into account in elastic energy.

\subsubsection{Work of external forces}

Considering the distribution of external forces per unit length of the beam ( $\left.f_{\text {external }}\right)$, the work by the external 
forces can be obtained as:

$$
V_{\text {external }}=\int_{0}^{L} f_{\text {external }} W(X) d X .
$$

The external force, $f_{\text {external }}$, is the summation of electrochemical and dispersion forces. The electrochemical force, $F_{E}$, is the sum of the electrical force, $F_{e}$, and chemical (or osmotic) force, $F_{c}$ :

$$
F_{E}=F_{e}+F_{c}
$$

For small potentials, $F_{e}$ and $F_{c}$ can be approximated as [14]:

$$
\begin{aligned}
& F_{c}=\frac{n_{\infty} e^{2} z_{0}^{2} A}{K_{B} T} \psi^{2}, \\
& F_{e}=-\frac{n_{\infty} e^{2} z_{0}^{2} A}{K_{B} T}\left(\frac{d \psi}{d Z}\right)^{2},
\end{aligned}
$$

where $K_{B}$ is the Boltzmann constant, $n_{\infty}$ is the bulk concentration, $T$ is the absolute temperature, $e$ is the electronic charge, $z_{0}$ is the absolute value of the valence, $\psi$ is the electric potential of the liquid-immersed electrodes (which is the summation of applied potential and zeta potential for each electrode), and $A$ is the electrode area.

The electrical potential, $\psi$, is determined by solving Eq. (17), i.e. the linear Poisson-Boltzmann equation (the Debye-Hückel approximation) [14]:

$$
\frac{d^{2} \psi}{d Z^{2}}=\kappa^{2} \psi, \quad \psi(Z=0)=\psi_{1}, \quad \psi(Z=g)=\psi_{2},
$$

where $g$ is the initial gap between two electrodes and $1 / \kappa=\varepsilon \varepsilon_{0} K_{B} T / 2 e^{2} z_{0}^{2} n_{\infty}$ is the Debye length. By solving Eq. (17) and substituting it in Eq. (13), the electrochemical force is obtained as:

$$
\begin{aligned}
F_{E}= & \frac{b \varepsilon \varepsilon_{0} \kappa^{2} \psi_{1}^{2}}{2 \sinh ^{2}(\kappa(g-W(X)))} \\
& \left(2 \frac{\psi_{2}}{\psi_{1}} \cosh (\kappa(g-W(X)))-\left[1+\left(\frac{\psi_{2}}{\psi_{1}}\right)^{2}\right]\right),
\end{aligned}
$$

where $\varepsilon_{0}$ is the permittivity of vacuum and $\varepsilon$ is the relative permittivity of the dielectric medium. The electrochemical force can be attractive or repulsive, depending on the parameters.

The dispersion forces per unit length of the beam $\left(f_{\text {disp }}\right)$ are defined considering the van der Waals and Casimir force regimes. Based on what is mentioned, two interaction regimes can be defined: first, the large separation regime in which the Casimir force is dominant (typically above several tens of nanometers [64$66])$.
The Casimir energy due to a quantum field is the sum of the zero point energies of the quantum field [67]. We assume that the electrodes are made from fully conductive materials; therefore, when the initial gap separation, $g$, is much smaller than the beam thickness, the leading terms of the interaction term of the Casimir force per unit length of beam are [67]:

$$
f_{\text {Cas }}=\frac{\pi^{2} \hbar b}{240 \sqrt{\tau v}(g-W(X))^{4}},
$$

where $\hbar=1.055 \times 10^{-34}$ Js is Planck's constant divided by $2 \pi$, and $\tau$ and $\nu$ are the permittivity and the permeability of fluid, respectively.

The second regime is the small separation regime (typically below several tens of nanometers [64-66]), in which the van der Waals force is the dominant attraction. In this case, the attraction between two ideal surfaces is proportional to the inverse cube of the separation:

$$
f_{\mathrm{vdW}}=\frac{A_{H} b}{6 \pi(g-W(X))^{3}},
$$

where $A_{H}$ is the Hamaker constant.

\subsubsection{Dimensionless relation for total energy}

By using Eqs. (11) and (12), the total energy of system can be summarized as:

$$
\begin{aligned}
\Pi= & \frac{1}{2} \int_{0}^{L}\left[\left(E I+2 \mu A l_{0}^{2}+\frac{8}{15} \mu A l_{1}^{2}+\mu A l_{2}^{2}\right)\left(\frac{d^{2} W}{d X^{2}}\right)^{2}\right. \\
& \left.+A\left(2 \mu l_{0}^{2}+\frac{4}{5} \mu l_{1}^{2}\right)\left(\frac{d^{3} W}{d X^{3}}\right)^{2}\right] d X \\
& -\int_{0}^{L} f_{\text {external }} W(X) d X
\end{aligned}
$$

Now, by using the substitutions $x=X / L$ and $w=$ $W / g$, the nondimensional total energy can be explained as:

$$
\begin{aligned}
\bar{\Pi}= & \frac{1}{2} \int_{0}^{1}\left[\left(1+\frac{\mu_{s}}{15}\left(30\left(\frac{l_{0}}{l_{2}}\right)^{2}+8\left(\frac{l_{1}}{l_{2}}\right)^{2}+15\right)\right)\left(\frac{d^{2} w}{d x^{2}}\right)^{2}\right. \\
& \left.+\frac{\mu_{s}}{30(L / h)^{2}}\left(5\left(\frac{l_{0}}{l_{2}}\right)^{2}+2\left(\frac{l_{1}}{l_{2}}\right)^{2}\right)\left(\frac{d^{3} w}{d x^{3}}\right)^{2}\right] d x \\
& -\int_{0}^{1}\left(\frac{\alpha_{n}}{(1-w)^{n}}-\frac{\beta^{2}}{\sinh ^{2}\left(\xi_{0}(1-w)\right)}\right. \\
& \left.\left\{\lambda \cosh \left(\xi_{0}(1-w)\right)-\frac{1}{2}\left(1+\lambda^{2}\right)\right\}\right) w(x) d x
\end{aligned}
$$


where the dimensionless parameters are identified as:

$$
\begin{aligned}
& \alpha_{n}=\left\{\begin{array}{l}
\frac{\bar{A} b L^{4}}{6 \pi g^{4} E I} \\
\frac{\pi^{2} \hbar b L^{4}}{240 \sqrt{\tau v} g^{5} E I}
\end{array}\right. \\
& \beta=\psi_{1} \sqrt{\frac{b \varepsilon \varepsilon_{0} \kappa^{2} L^{4}}{g}}, \\
& \gamma=\frac{g}{b}, \\
& \mu_{s}=\frac{12 \mu}{E\left(h / l_{2}\right)^{2}}, \\
& \lambda=\frac{\psi_{2}}{\psi_{1}} \\
& \xi_{0}=\kappa g .
\end{aligned}
$$$$
\text { vdW interaction }(n=3)
$$$$
\text { Casimir interaction }(n=4)(23 \mathrm{a})
$$

In the above relations, $\beta, \mu_{s}, \alpha_{n}$, and $\xi_{0}$ interpret the dimensionless values of beam electrode voltage, sizeeffect, dispersion forces, and bulk ion concentration, respectively. The dimensionless parameter indicates the ratio of potential on the ground electrode over the beam electrode.

It should be noted that Relations (23) turns into that of the classical theory by setting $l_{0}, l_{1}$, and $l_{2}$ equal to zero. Furthermore, the size-dependent behavior of nano-beam based on the modified couple stress theory can be obtained by considering $l_{0}=l_{1}=0$ and $l_{2}=l$.

\section{Solution methods}

\subsection{Rayleigh-Ritz method}

To solve the governing equation of the systems, the displacement is expressed as a linear combination of a complete set of independent basis functions $\varphi_{i}(x)$ in the form of:

$$
w(x)=\sum_{i=1}^{n} q_{i} \phi_{i}(x),
$$

where the index $i$ refers to the number of terms included in the simulation. We use the free vibration mode shapes of the nano-beam as basic functions in the Rayleigh-Ritz procedure. The mode shapes of cantilever nano-beam can be expressed as:

$$
\begin{aligned}
& \phi_{i}(\xi)=\cosh \left(\lambda_{i} \xi\right)-\cos \left(\lambda_{i} \xi\right) \\
& \quad-\frac{\cosh \left(\lambda_{i}\right)-\cos \left(\lambda_{i}\right)}{\sinh \left(\lambda_{i}\right)-\sin \left(\lambda_{i}\right)}\left(\sinh \left(\lambda_{i} \xi\right)-\sin \left(\lambda_{i} \xi\right)\right),
\end{aligned}
$$

where $\lambda_{i}$ is the $i$ th root of the characteristic equation of clamped-free beams. At the equilibrium point, the following relation must be satisfied:

$$
\frac{\partial \bar{\Pi}}{\partial q_{i}}=0 \quad i=0,1, \cdots, N .
$$

This led to a system of algebraic equation which could be solved numerically to obtain the final solution. By using Taylor expansion for electrostatic and dispersion forces, substituting Eqs. (24) and (25) into Eq. (26), assuming the orthogonality of $\varphi_{i}(x)$, and then following some straightforward mathematical operations, a system of algebraic equation can be defined as:

$$
\begin{aligned}
& {\left[1+\frac{\mu_{s}}{15}\left(30\left(\frac{l_{0}}{l_{2}}\right)^{2}+8\left(\frac{l_{1}}{l_{2}}\right)^{2}+15\right)\right] \lambda_{i}^{4} q_{i}} \\
& -\frac{\mu_{s}}{30(L / h)^{2}}\left[5\left(\frac{l_{0}}{l_{2}}\right)^{2}+2\left(\frac{l_{1}}{l_{2}}\right)^{2}\right] \\
& \int_{0}^{1}\left(\sum_{j=1}^{N} q_{j} \frac{d^{6}}{d X^{6}} \phi_{j}\right) \phi_{i} d x \\
& -\int_{0}^{1} \phi_{i} \sum_{k=0}^{\infty} A_{k}\left(\sum_{j=1}^{N} q_{j} \phi_{j}\right)^{k} d x+B . C .=0 \\
& i=1,2, \cdots, N
\end{aligned}
$$

where $N$ is the number of considered terms of RayleighRitz and $A_{k}$ is the Taylor expansion coefficient of electrostatic and dispersion forces. In the above equation, the Boundary Condition terms, B.C., are defined as:

$$
\begin{aligned}
\text { B.C. }= & \frac{\mu_{s}}{30(L / h)^{2}}\left(5\left(\frac{l_{0}}{l_{2}}\right)^{2}+2\left(\frac{l_{1}}{l_{2}}\right)^{2}\right) \\
& \left.\left(\sum_{j=1}^{N} q_{j} \frac{d^{3}}{d X^{3}} \phi_{j}\right) \frac{d^{2} \phi_{i}}{d X^{2}}\right|_{x=1} \\
& -\frac{\mu_{s}}{30(L / h)^{2}}\left(5\left(\frac{l_{0}}{l_{2}}\right)^{2}+2\left(\frac{l_{1}}{l_{2}}\right)^{2}\right) \\
& \left.\left(\sum_{j=1}^{N} q_{j} \frac{d^{3}}{d X^{3}} \phi_{j}\right) \frac{d^{2} \phi_{i}}{d X^{2}}\right|_{x=0} \\
& +\frac{\mu_{s}}{30(L / h)^{2}}\left(5\left(\frac{l_{0}}{l_{2}}\right)^{2}+2\left(\frac{l_{1}}{l_{2}}\right)^{2}\right) \\
& \left.\left(\sum_{j=1}^{N} q_{j} \frac{d^{5}}{d X^{5}} \phi_{j}\right) \phi_{i}\right|_{x=1}
\end{aligned}
$$




$$
\begin{aligned}
& -\frac{\mu_{s}}{30(L / h)^{2}}\left(5\left(\frac{l_{0}}{l_{2}}\right)^{2}+2\left(\frac{l_{1}}{l_{2}}\right)^{2}\right) \\
& \left.\left(\sum_{j=1}^{N} q_{j} \frac{d^{4}}{d X^{4}} \phi_{j}\right) \frac{d \phi_{i}}{d X}\right|_{x=1} .
\end{aligned}
$$

The Maple commercial software is employed to numerically solve the system of equations.

The instability occurs when $d w(x=1) / d \beta^{2} \rightarrow 0$. The instability parameters of the system can be determined via the slope of the $w-\beta$ graphs by plotting $w$ versus $\beta$.

\subsection{Numerical solution method}

In addition to the analytical method, the deflection of the nano-cantilever is numerically simulated using Maple software. For this purpose, the governing differential equation of the system is required. Utilizing Hamilton principle, i.e. $\delta(\bar{\Pi})=0$, in which $\delta$ indicates variations symbol, the governing equation of lateral deflection of the system can be derived as the following:

$$
\begin{gathered}
{\left[1+\frac{\mu_{s}}{15}\left(30\left(\frac{l_{0}}{l_{2}}\right)^{2}+8\left(\frac{l_{1}}{l_{2}}\right)^{2}+15\right)\right] \frac{\partial^{4} w}{\partial x^{4}}} \\
-\frac{\mu_{s}}{30(L / h)^{2}}\left(5\left(\frac{l_{0}}{l_{2}}\right)^{2}+2\left(\frac{l_{1}}{l_{2}}\right)^{2}\right) \frac{\partial^{6} w}{\partial x^{6}} \\
=\frac{\alpha_{n}}{(1-w)^{n}}-\frac{\beta}{\sinh ^{2}\left(\xi_{0}(1-w)\right)} \\
\quad\left\{\lambda \cosh \left(\xi_{0}(1-w)\right)-\frac{1}{2}\left(1+\lambda^{2}\right)\right\} .
\end{gathered}
$$

With the following boundary conditions:

$$
\begin{aligned}
& w(0)=\frac{d w}{d x}(0)=\frac{d^{3} w}{d x^{3}}(0)=0, \\
& {\left[1+\mu_{s}\left(2\left(\frac{l_{0}}{l_{2}}\right)^{2}+\frac{8}{15}\left(\frac{l_{1}}{l_{2}}\right)^{2}+1\right)\right] \frac{d^{3} w}{d x^{3}}(1)}
\end{aligned}
$$

$$
\begin{aligned}
& -\frac{\mu_{s}}{30(L / h)^{2}}\left(5\left(\frac{l_{0}}{l_{2}}\right)^{2}+2\left(\frac{l_{1}}{l_{2}}\right)^{2}\right) \frac{d^{5} w}{d x^{5}}(1)=0 \\
& {\left[1+\mu_{s}\left(2\left(\frac{l_{0}}{l_{2}}\right)^{2}+\frac{8}{15}\left(\frac{l_{1}}{l_{2}}\right)^{2}+1\right)\right] \frac{d^{2} w}{d x^{2}}(1)} \\
& -\frac{\mu_{s}}{30(L / h)^{2}}\left(5\left(\frac{l_{0}}{l_{2}}\right)^{2}+2\left(\frac{l_{1}}{l_{2}}\right)^{2}\right) \frac{d^{4} w}{d x^{4}}(1)=0 \\
& \frac{d^{3} w}{d x^{3}}(1)=0 .
\end{aligned}
$$

By numerically solving the differential equations, the deflections of the nano-cantilever are determined. When instability occurs, no solution exists for Eq. (30) and the pull-in parameters of the system can be determined by plotting the NEMS tip deflection versus the applied force.

\section{Result and discussion}

In the following, typical nano-actuator with the geometrical characteristics of $h / L=20$ and $\lambda=0.1$ is considered. The Young's modulus $E$ and shear modulus $\mu$ are $169 \mathrm{GPa}$ and $65.8 \mathrm{GPa}$, respectively.

\subsection{Verification}

To validate the RAM and check the convergence rate of series, effect of increasing the number of modes on deflection of typical NEMS is presented in Table 1. This table shows the variations of tip deflection of the typical NEMS considering various series terms for $\alpha_{3}=0.5, \beta=1$, and $l_{0}=l_{1}=l_{2}=5 h$. This table reveals the convergence of the series with increasing the number of modes. By selecting only one mode, an acceptable error is achieved. Therefore, one mode is considered to determine instability of the NEMS.

Table 2 shows the variation of tip deflection of the typical NEMS considering various Taylor expansion series terms for $\alpha_{3}=0.5$ and $l_{0}=l_{1}=l_{2}=5 h$. The selected voltage equals $0.9 \beta_{\mathrm{PI}}$. This table reveals the

Table 1. The convergence check of RRM reduced order method.

\begin{tabular}{lcccc}
\hline & \multicolumn{3}{c}{ RRM } & \multirow{2}{*}{ Numerical } \\
\cline { 2 - 5 } & 1 term & 2 terms & 3 terms & \\
\hline $\boldsymbol{w}(\boldsymbol{x}=\mathbf{1})$ & 0.04698 & 0.046416 & 0.046424 & 0.046457 \\
Related error (\%) & 1.124458 & 0.089038 & 0.07206 & - \\
\hline
\end{tabular}

Table 2. The convergence check of Taylor expansion series.

\begin{tabular}{lccccc}
\hline & \multicolumn{2}{c}{ Number of Taylors series terms in RRM } & \multirow{2}{*}{ Numerical } \\
\cline { 2 - 5 } & $\mathbf{2}$ terms & $\mathbf{3}$ terms & 4 terms & 5 terms & \\
\hline $\boldsymbol{w}(\boldsymbol{x}=\mathbf{1})$ & 0.191332 & 0.229715 & 0.250006 & 0.26248 & 0.259441 \\
Related error (\%) & 26.3 & 11.5 & 3.6 & 1.2 & - \\
\hline
\end{tabular}




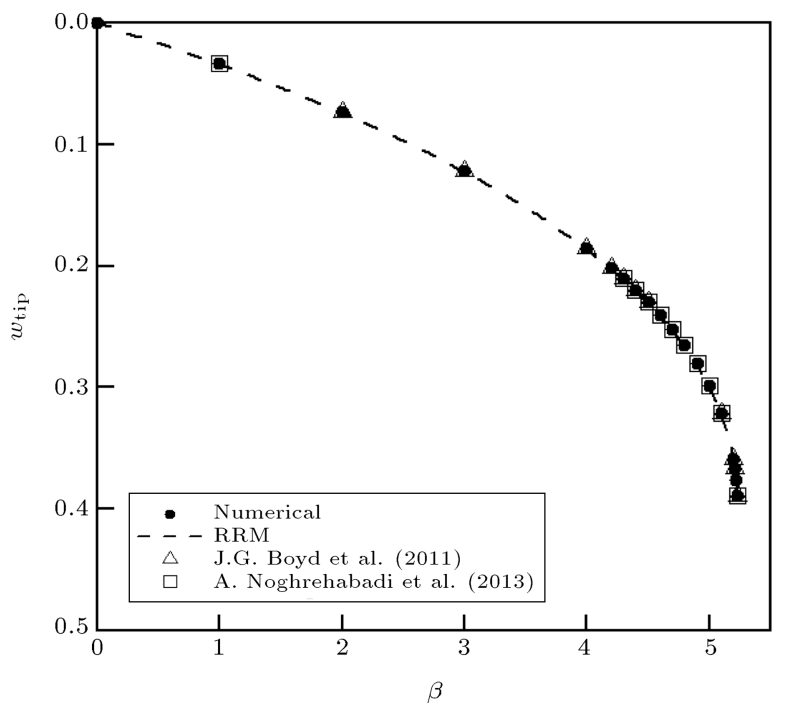

Figure 2. Variation of the normalized tip deflection as a function of applied voltage. The present approach is in very good agreement with literature.

convergence of the series with increasing the number of Taylor expansion terms.

To validate the present theoretical model with literature, the governing equation of nano-actuator was solved based on classical theory $\left(l_{0}=l_{1}=l_{2}=0\right)$ and neglecting dispersion forces. Figure 2 shows the influences of the applied voltage, $\beta$, on the normalized tip deflection, $w(x=1)$, of the cantilever beam for $\alpha_{n}=0, \lambda=0.1$, and $\xi_{0}=1$. It can be observed that the normalized tip deflection would increase with an increase in the input voltage. This figure reveals that the present approach is in very good agreement with the finite element solution [14] and modified Adomian results [15].

\subsection{NEMS deflection and pull-in instability}

Figure 3 shows the variation of deflection of typical cantilever NEMS when the applied voltage increases from zero to pull-in value. In this figure, the vertical axis reveals the deflection of the nano-beams while the horizontal axis reveals the dimensionless length of the beams.

The dimensionless material length-scale parameters $l_{0} / l_{2}, l_{1} / l_{2}$, and $h / l_{2}$ are equally selected as 1 ; furthermore, the dispersion force parameter is considered equal to $0.2\left(\alpha_{n}=0.2\right)$ and $\xi_{0}=1.5$. As seen, increasing the applied voltage increases the deflection of the nano-beam. When the applied voltage exceeds its critical value, $\beta_{\mathrm{PI}}$, no solution exists and the pull-in instability occurs. Note that the operation distance of the nano-systems is limited by this instability. This figure shows that the nano-beam has an initial deflection when no voltage is applied, which is due to dispersion forces. It is shown that the results of RRM are in good agreement with those of the numerical

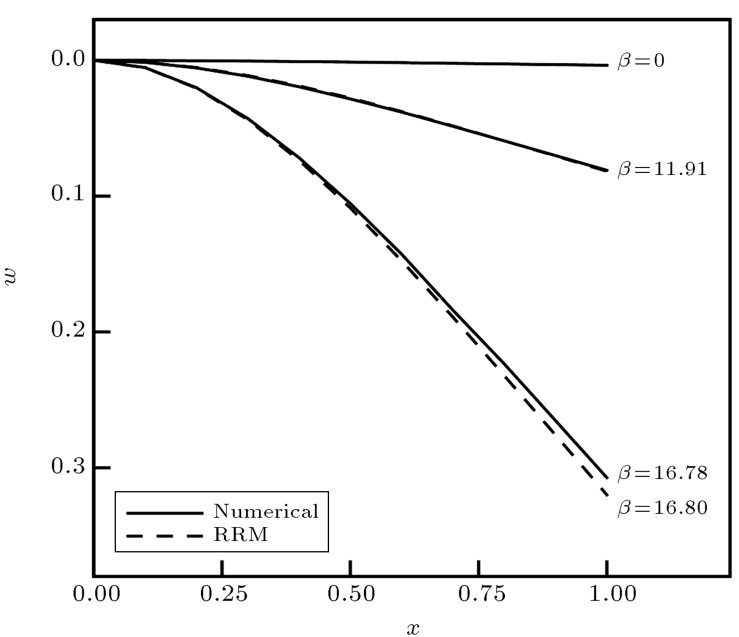

(a) vdW force

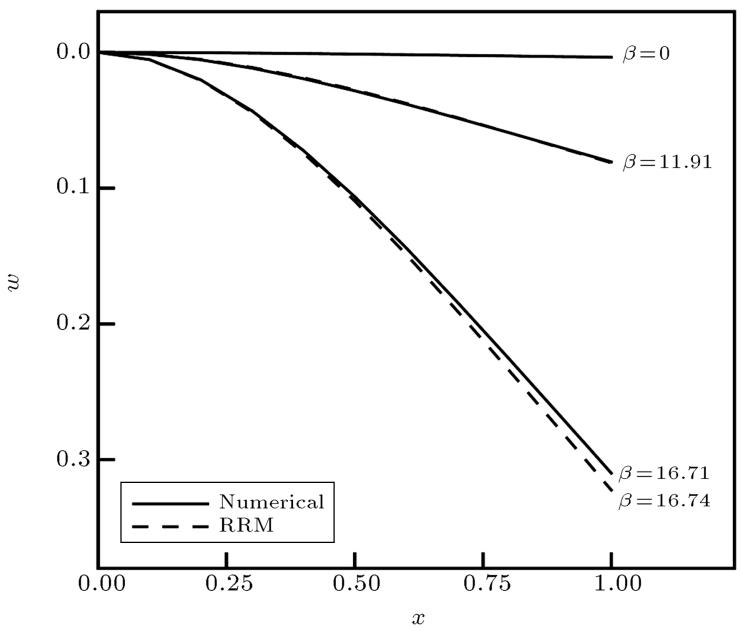

(b) Casimir force

Figure 3. Deflection of the cantilever NEMS for different values of applied voltage from 0 to pull-in voltage.

method. The relative error of the presented methods with respect to the numerical solution is less that $2 \%$.

\subsection{Influence of size effect}

Variation of the pull-in voltage $\left(\beta_{\mathrm{PI}}\right)$ of the nano-beams is demonstrated in Figure 4 as a function of the nanoscale parameter $\left(h / l_{2}\right)$. The horizontal lines correspond to the pull-in voltage $\left(\beta_{\mathrm{PI}}\right)$ when no size-effect has been considered (i.e., classical theory). This figure shows that decreasing $h / l_{2}$ results in decreasing the pull-in voltage of the nano-system. It should be noted that decrease in $h / l_{2}$ value corresponds to increase in size-effect. This means that size-effect provides a hardening behavior that enhances the elastic resistance and consequent pull-in voltage of the nano-device. On the other hand, with increase in the beam thickness, results of strain gradient theory approach those of the classic continuum theory, i.e. enhancing the beam thickness decreases the size-effect. 


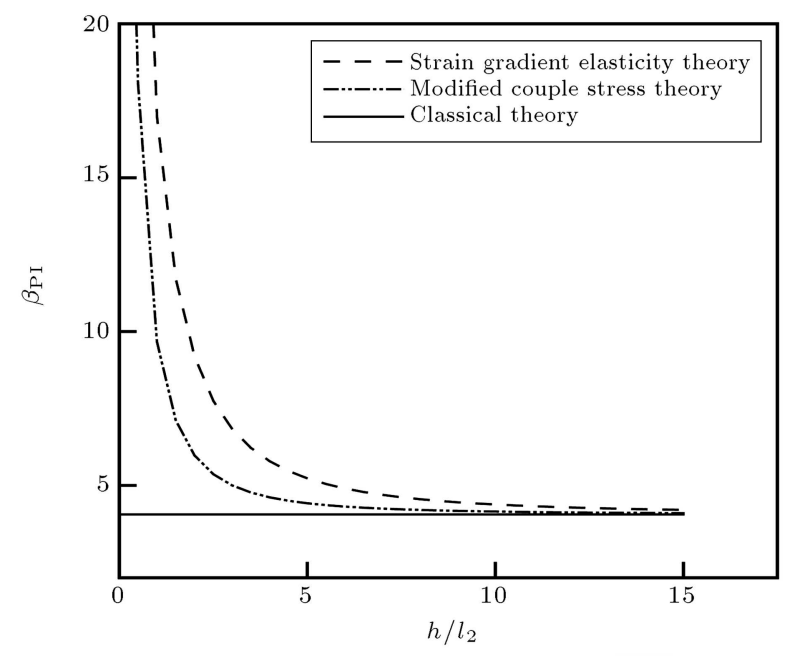

(a) Numerical solution

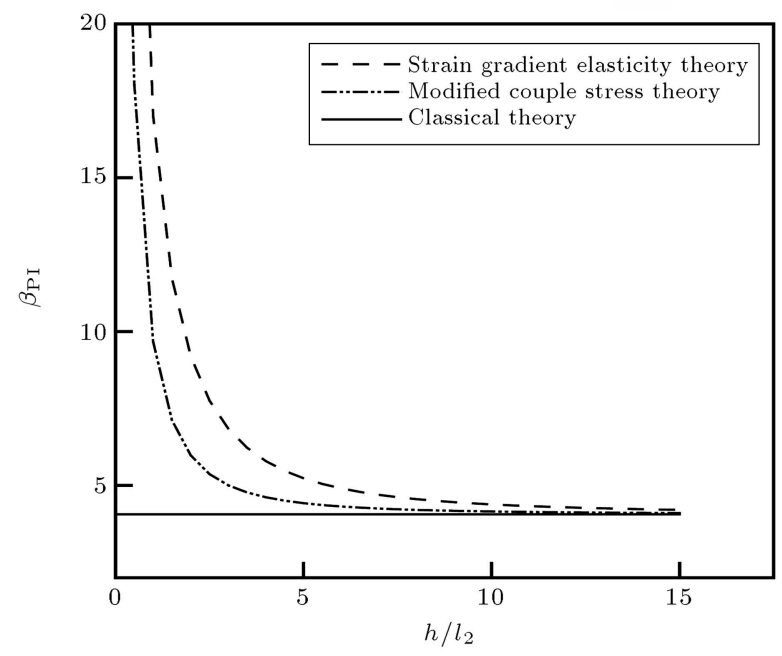

(b) RRM

Figure 4. Pull-in voltage for different models as a function of size effect parameter.

Figure 5 represents the influence of size-effect $\left(h / l_{2}\right)$ on the instability deflection $\left(w_{\mathrm{PI}}\right)$ of the nanocantilever. This figure shows that the pull-in deflection predicted using the classical and modified couple stress theories is independent on the size-effect. However, the value of pull-in deflection determined by strain gradient theory slightly decreases by increasing the length-scale parameter.

\subsection{Influence of dispersion forces}

If the gap between the cantilever and the ground is of the order of several nanometers, the effect of dispersion forces must be taken into account. The effect of dispersion forces on pull-in voltage of the NEMS is presented in Figure 6. As shown, increasing the dispersion forces leads to a decrease in the pullin voltage of the nano-cantilever. Interestingly, the intersection point of the curves and the horizontal axis correspond to the critical value of dispersion forces in liquid media. When the nano-cantilever

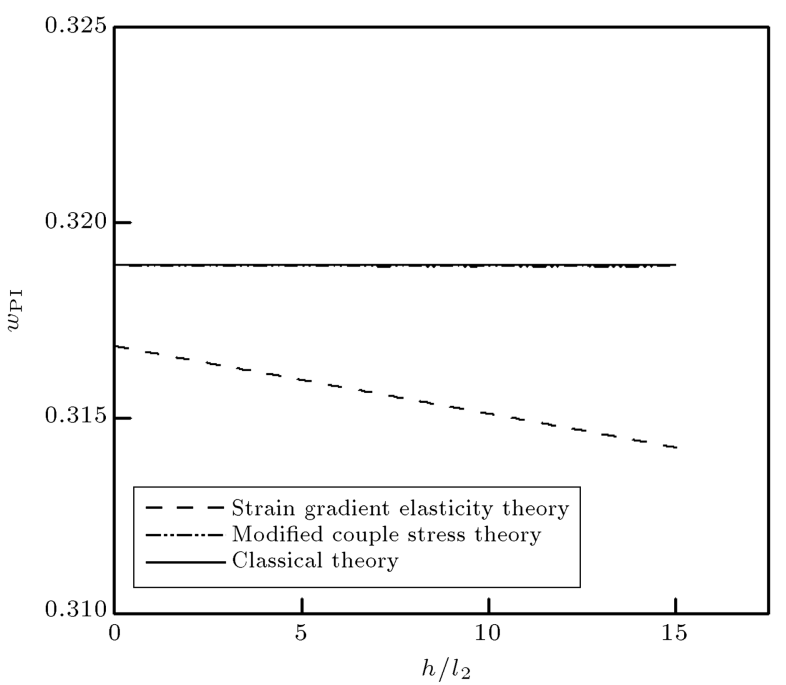

(a) Numerical solution

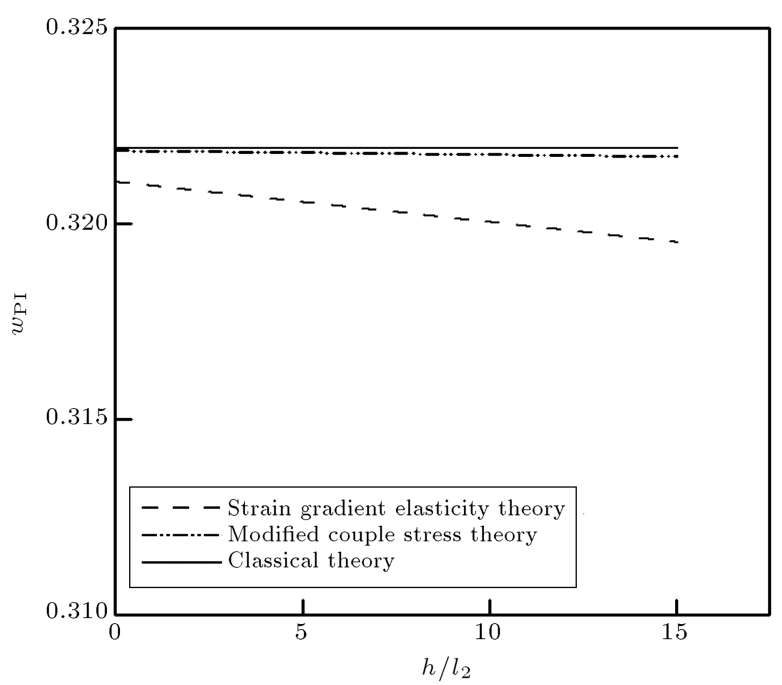

(b) RRM

Figure 5. Pull-in deflection for different models as a function of size effect parameter.

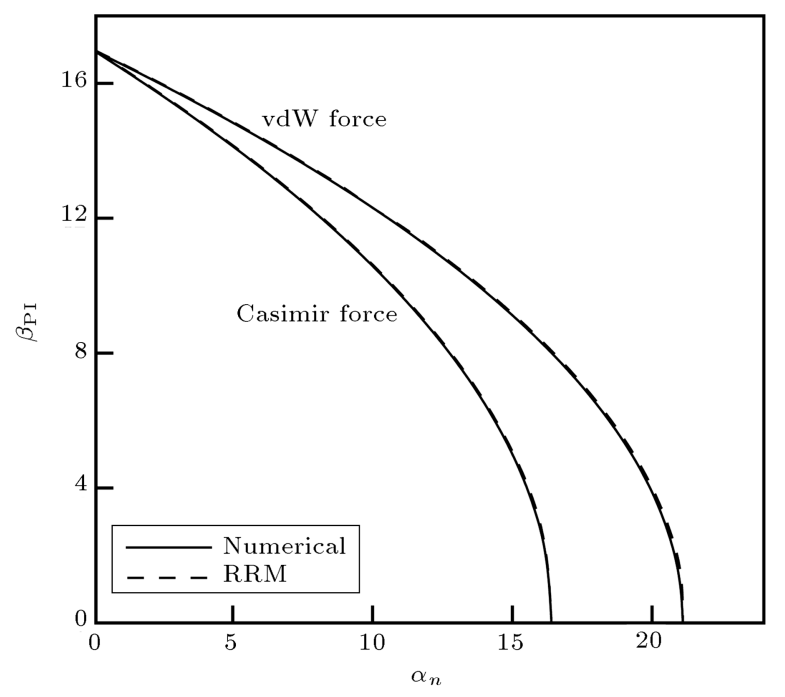

Figure 6. Effect of dispersion force on pull-in voltage. 


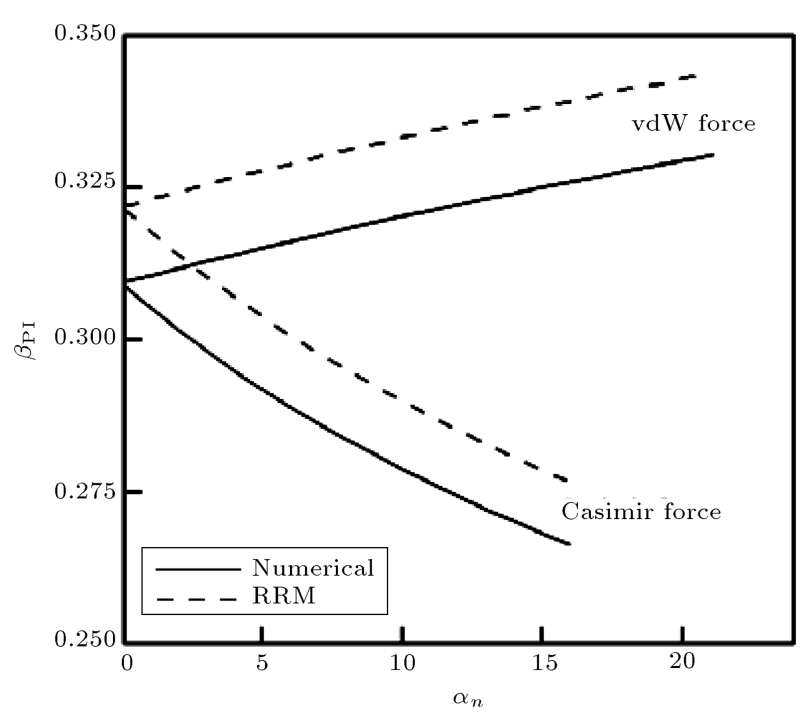

Figure 7. Effects of dispersion forces on pull-in deflection.

is close enough to the ground, dispersion forces can induce stiction even without any electrostatic force. Influence of dispersion on the pull-in deflection of the system is presented in Figure 7 . This figure shows that while increase in the van der Waals force slightly reduces the pull-in deflection of the NEMS, increasing the Casimir traction increases the pull-in deflection of the NEMS. It is worth to note that the effect of dispersion forces on the pull-in voltage of the liquid-immersed beam-type structure is similar to that reported for non-immersed nanostructures $[28,68]$. It is reported that the dispersion forces reduce the pullin voltage of torsional systems as well as beam-type ones $[28,68]$.

\subsection{Effects of ion concentration}

Variation of the pull-in voltage versus ion concentration parameter $\left(\xi_{0}\right)$ is shown in Figure 8 considering the presence of dispersion forces. This figure illustrates the effect of ion concentration on the instability of nanocantilever. Augmentation of ions in the vicinity of the surface of electrodes increases the pull-in voltage. Figure 9 illustrates the effect of ion concentration on the pull-in deflection of nano-cantilever. As seen, increasing ion concentration can decrease pull-in deflection. These figures reveal that increase in the Debye length of the electrolyte enhances the pull-in voltage while it can reduce the pull-in deflection of the system.

\section{Conclusion}

In this article, strain gradient theory has been employed to investigate the size-dependent pull-in performance of cantilever NEMS immersed in liquid electrolytes incorporating the effect of dispersion forces. The governing non-linear equation was solved using

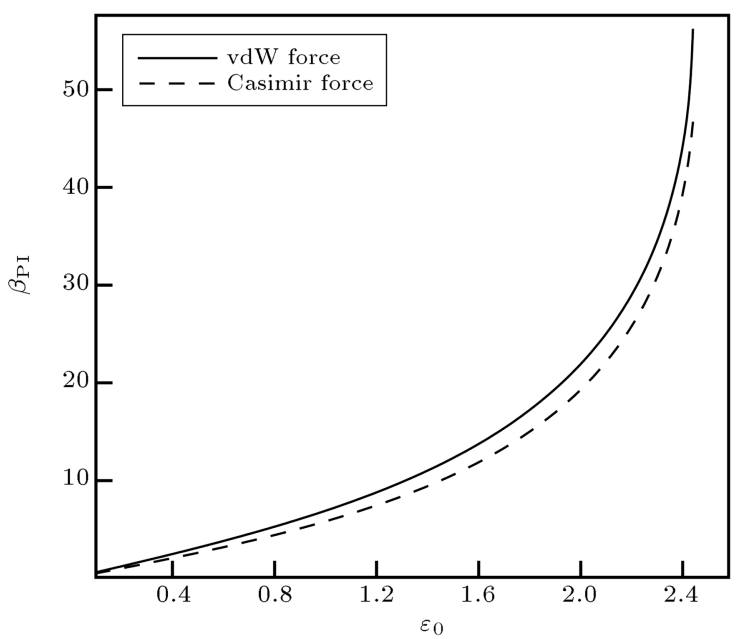

(a) Numerical solution

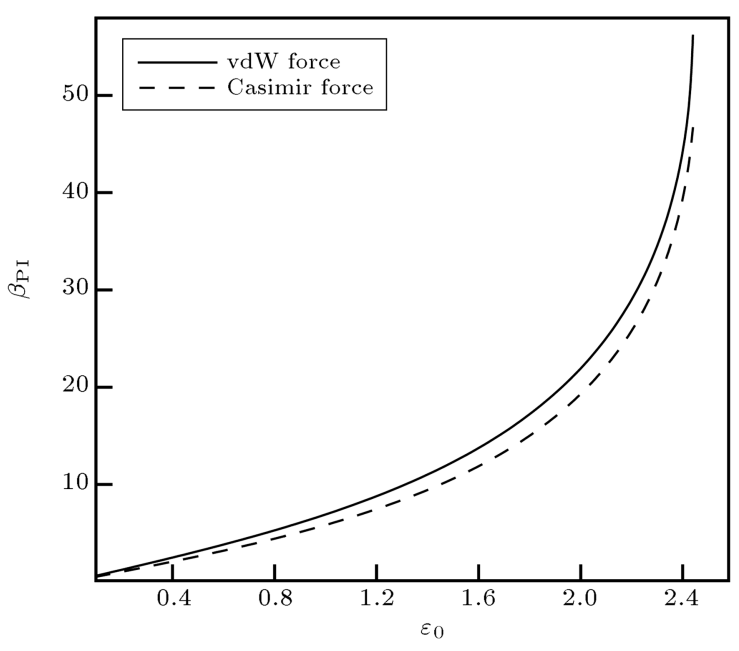

(b) RRM

Figure 8. Effect of ion concentration on the pull-in voltage for $\alpha_{n}=10$.

different approaches, i.e. RRM, and numerical method. Comparison between solving methods reveals that RRM is in good agreement with numerical solution. It is found that:

- The presence of dispersion force reduces pull-in voltage of the system. While increase in Casimir force reduces the pull-in deflection of the cantilever NEMS, enhancing the van der Waals attraction increase the instability deflection of the system;

- For ultra-thin NEMS where the beam thickness is comparable with the material length-scale parameters, there is a substantial difference between the results of classic continuum theory and those of strain gradient and modified couple stress theories. Size-effect increases the pull-in voltage of the NEMS due to the stiffness effect;

- Augmentation of bulk ion concentration increases the pull-in voltage of the liquid-immersed cantilever 


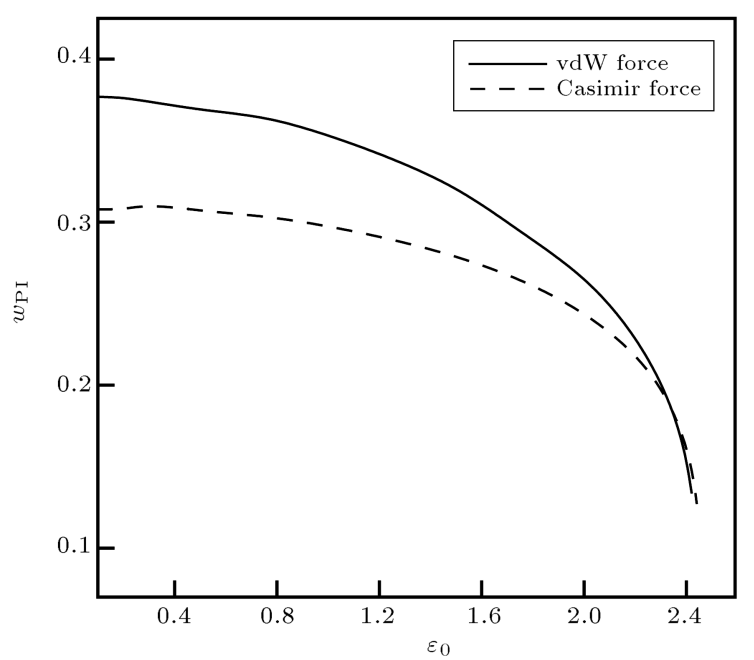

(a) Numerical solution

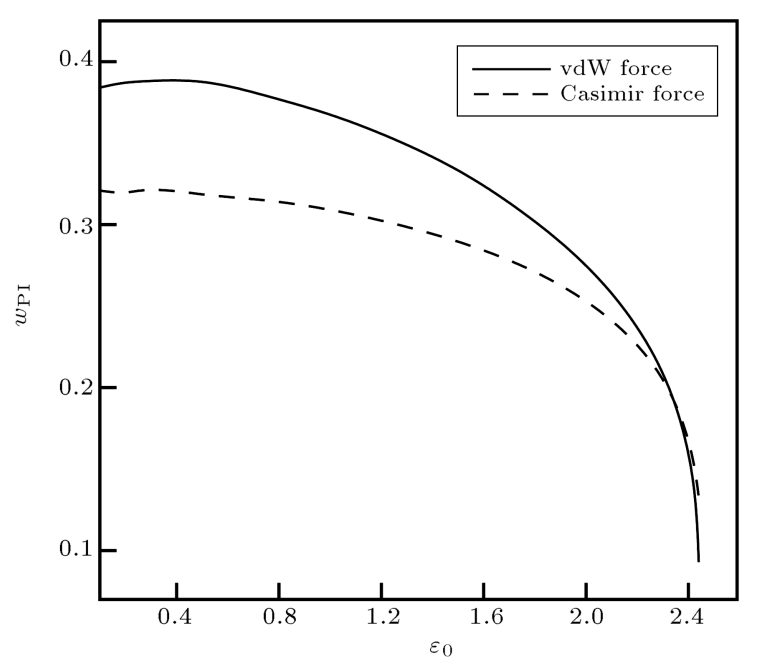

(b) RRM

Figure 9. Effects of ion concentration on the pull-in deflection.

NEMS. However, it can decrease the pull-in deflection of the system.

\section{References}

1. Ke, C.H. and Espinosa, H.D. "Nanoelectromechanical systems (NEMS) and modeling", in Handbook of Theoretical and Computational Nanotechnology, M. Rieth, W. Schommers and P.D. Gennes, Eds., American Scientific Publishers (2006).

2. Sedighi, H.M. and Shirazi, K.H. "Vibrations of microbeams actuated by an electric field via parameter expansion method", Acta Astronautica, 85, pp. 19-24 (2013).

3. Rezazadeh, Gh., Fathalilou, M. and Sadeghi, M. "Pullin voltage of electrostatically-actuated microbeams in terms of lumped model pull-in voltage using novel design corrective coefficients", Sensing and Imaging: An International Journal, 12(3-4), pp. 117-131 (2011).
4. Batra, R.C., Porfiri, M. and Spinello, D. "Capacitance estimate for electrostatically actuated narrow microbeams", Micro \& Nano Letters, IET, 1(2), pp. 71-73 (2006).

5. Rollier, S., Legrand, B., Collard, D. and Buchaillot, L. "The stability and pull-in voltage of electrostatic parallel-plate actuators in liquid solutions", J. Micromech. Microeng., 16, pp. 794-801 (2006).

6. Loh, O., Vaziri, A. and Espinosa, H.D. "The potential of MEMS for advancing experiments and modeling in cell mechanics", Experimental Mechanics, 49, pp. 105124 (2009).

7. Oh, K., Chung, J.H., Devasia, S. and Riley, J.J. "Biomimetic silicone cilia for microfluidic manipulation", Lab on a Chip, 9(11), pp. 1561-1566 (2009).

8. Tsai, N.C. and Sue, C.Y. "Review of MEMS-based drug delivery and dosing systems", Sensors and Actuators A, 134, pp. 555-564 (2007).

9. Boyd, J.G. and Kim, D. "Nanoscale electrostatic actuators in liquid electrolytes", Journal of Colloid and Interface Science, 301, pp. 542-548 (2006).

10. Gramse, G., Edwards, M.A., Fumagalli, L. and Gomila, G. "Dynamic electrostatic force microscopy in liquid media", Applied Physics Letters, 101(21), 213108 (2012).

11. Rezazadeh, Gh. Fathalilou, M., Shabani, R. Tarverdilou, S. and Talebian, S. "Dynamic characteristics and forced response of an electrostatically-actuated microbeam subjected to fluid loading", Microsyst Technol., 15, pp. 1355-1363 (2009).

12. Sounart, T.L., Michalske, T.A. and Zavadil, K.R. "Frequency-dependent electrostatic actuation in microfluidic MEMS", J. Microelectromech. Syst., 14(1), pp. 125-133 (2005).

13. Maali, A., Hurth, C., Boisgard, R., Jai, C., Touria, C.B., and Aimé, J.P. "Hydrodynamics of oscillating atomic force microscopy cantilevers in viscous fluids", Journal of Applied Physics, 97(7), 074907 (2005).

14. Boyd, J.G. and Lee, J. "Deflection and pull-in instability of nanoscale beams in liquid electrolytes", Journal of Colloid and Interface Science, 356, pp. 387-394 (2011).

15. Noghrehabadi, M., Eslami, M. and Ghalambaz, M. "Influence of size effect and elastic boundary condition on the pull-in instability of nano-scale cantilever beams immersed in liquid electrolytes", International Journal of Non-Linear Mechanics, 52, pp. 73-78 (2013).

16. Yang, C.Y. and Zhao, Y.P. "Influences of hydration force and elastic strain energy on the stability of solid film in a very thin solid-on-liquid structure", The Journal of Chemical Physics, 120(11), pp. 5366-5376 (2004).

17. Buks, E. and Roukes, M.L. "Stiction, adhesion energy, and the Casimir effect in micromechanical systems", Phys. Rev. B., 63, 033402 (2001). 
18. Buks, E. and Roukes, M.L. "Metastability and the Casimir effect in micromechanical systems", Europhys. Lett., 54, pp. 220-226 (2001).

19. Ramezani, A., Alasty, A. and Akbari, J. "Analytical investigation and numerical verification of Casimir effect on electrostatic nano-cantilevers", Microsyst. Technol., 14, pp. 145-157 (2008).

20. Ramezani, A., Alasty, A. and Akbari, J. "Closed-form solutions of the pull-in instability in nano-cantilevers under electrostatic and intermolecular surface forces", Int. J. Solids Struct., 44, pp. 4925-4941 (2007).

21. Farrokhabadi, A., Rach, R. and Abadyan, M.R. "Modeling the static response and pull-in instability of CNT nanotweezers under the Coulomb and van der Waals attractions", Physica E: Low-dimensional Systems and Nanostructures, 53, pp. 137-145 (2013).

22. Tadi Beni, Y., Koochi, A. and Abadyan, M.R. "Theoretical study of the effect of Casimir force, elastic boundary conditions and size dependency on the pullin instability of beam-type NEMS", Physica E., 43, pp. 979-988 (2011).

23. Noghrehabadi, A.R., Tadi Beni, Y., Koochi, A., Sadat Kazemi, A.R., Yekrangi, A., Abadyan, M.R. and Noghreh Abadi, M. "Closed-form approximations of the pull-in parameters and stress field of electrostatic cantilever nano-actuators considering van der Waals attraction", Procedia Engineering, 10, pp. 3750-3756 (2011).

24. Koochi, A. and Abadyan, M.R. "Evaluating the ability of modified adomian decomposition method to simulate the instability of freestanding carbon nanotube: comparison with conventional decomposition method", Journal of Applied Sciences, 11, pp. 3421-3428 (2011).

25. Amirbostaghi, Gh., Khatibi, S., Hafshejani, M.K. and Arad, A. "Comparison between homotopy perturbation and modified Adomian decomposition methods in simulating the effect of van der Waals attraction in nano structures instability", World Applied Sciences Journal, 18(12), pp. 1813-1824 (2012).

26. Duan, J.S., Rach, R. and Wazwaz, A.M. "Solution of the model of beam-type micro- and nano-scale electrostatic actuators by a new modified Adomian decomposition method for nonlinear boundary value problems", Int. J. Non-Linear Mech., 49, pp. 159-169 (2013).

27. Johson, S.G. "Numerical methods for computing Casimir interactions", In Casimir Physics, Dalvit, D., Milonni, P., Roberts, D. and da Rosa, F., Eds., pp. 175-219, Springer (2011).

28. Guo, J.G. and Zhao, Y.P. "Influence of van der Waals and Casimir forces on electrostatic torsional actuators", Microelectromechanical Systems, Journal of, 13(6), pp. 1027-1035 (2004).

29. Lin, W.H. and Zhao, Y.P. "Nonlinear behavior for nanoscale electrostatic actuators with Casimir force", Chaos, Solitons \& Fractals, 23(5), pp. 1777-1785 (2005).
30. Lamoreaux, S.K. "The Casimir force: background, experiments, and applications", Reports on Progress in Physics, 68(1), p. 201 (2005).

31. Rotkin, S.V. "Analytical calculations for nanoscale electromechanical systems", Electromechanical Soc. Proc., 6, pp. 90-97 (2002).

32. Lin, W.H. and Zhao, Y.P. "Dynamic behavior of nanoscale electrostatic actuators", Chin. Phys. Lett., 20, pp. 2070-2073 (2003).

33. Abdi, J., Koochi, A., Kazemi, A.S. and Abadyan, M. "Modeling the effects of size dependency and dispersion forces on the pull-in instability of electrostatic cantilever NEMS using modified couple stress theory", Smart Mater. Struct., 20, 055011 (2011).

34. Soroush, R., Koochi, A., Kazemi, A.S., Abadyan, M. "Modeling the effect of van der Waals attraction on the instability of electrostatic cantilever and doubly-supported nano-beams using modified adomian method", International Journal of Structural Stability and Dynamics, 12, 1250036 (2012).

35. Dequesnes, M., Rotkin, S.V. and Aluru, N.R. "Calculation of pull-in voltages for carbon nanotube-based nanoelectromechanical switches", Nanotechnology, 13, pp. 120-131 (2002).

36. Kolpekwar, A., Kellen, C. and Blanton, R.D. "Fault model generation for MEMS", in Proceedings of the International Conference on Modeling and Simulation of Microsystems, Semiconductors, Sensors and Actuators, M. Laudon, B. Romanowicz, Eds., Computational Publications, Cambridge, MA, pp. 111-116 (1998).

37. Fleck, N.A., Muller, G.M., Ashby, M.F. and Hutchinson, J.W. "Strain gradient plasticity: theory and experiment", Acta Metall. Mater., 42, pp. 475-487 (1994).

38. Stolken, J.S. and Evans, A.G. "Microbend test method for measuring the plasticity length scale", Acta Mater., 46, pp. 5109-5115 (1998).

39. Lam, D.C.C., Yang, F., Chong, A.C.M., Wang, J. and Tong, P. "Experiments and theory in strain gradient elasticity", Journal of the Mechanics and Physics of Solids, 51(8), pp. 1477-1508 (2003).

40. McFarland, A.W. and Colton J.S. "Thermoplastic polymer microcantilever sensors fabricated via micromolding", J. Micromech. Microeng., 15, pp. 1060-1067 (2005).

41. Wang, W., Huang, Y., Hsia, K.J., Hu, K.X. and Chandra, A. "A study of microbend test by strain gradient plasticity", International Journal of Plasticity, 19(3), pp. 365-382 (2003).

42. McElhaney, K.W., Vlassak, J.J. and Nix, W.D. "Determination of indenter tip geometry and indentation contact area for depth-sensing indentation experiments", Journal of Materials Research, 13(05), pp. 1300-1306 (1998). 
43. Nix, W.D. and Huajian Gao "Indentation size effects in crystalline materials: a law for strain gradient plasticity", Journal of the Mechanics and Physics of Solids, 46(3), pp. 411-425 (1998).

44. Chong, A. and Lam, D.C.C. "Strain gradient plasticity effect in indentation hardness of polymers", Journal of Materials Research, 14(10), pp. 4103-4110 (1999).

45. Cao, Y., Nankivil, D.D., Allameh, S. and Soboyejo, W.O. "Mechanical properties of au films on silicon substrates", Materials and Manufacturing Processes, 22(2), pp. 187-194 (2007).

46. Abu Al-Rub, R.K. and Voyiadjis, G.Z. "Analytical and experimental determination of the material intrinsic length scale of strain gradient plasticity theory from micro-and nano-indentation experiments", International Journal of Plasticity, 20(6), pp. 1139-1182 (2004).

47. Maranganti, R. and Sharma, P. "A novel atomistic approach to determine strain-gradient elasticity constants: Tabulation and comparison for various metals, semiconductors, silica, polymers and the (ir) relevance for nanotechnologies", Journal of the Mechanics and Physics of Solids, 55(9), pp. 1823-1852 (2007).

48. Eringen, A.C. and Edelen, D.B.G. "On nonlocal elasticity", International Journal of Engineering Science, 10, pp. 233-248 (1972).

49. Kong, S. "Size effect on pull-in behavior of electrostatically actuated microbeams based on a modified couple stress theory", Applied Mathematical Modelling, 37, pp. 7481-7488 (2013).

50. Ejike, U.B.C.O. "The plane circular crack problem in the linearized couple-stress theory", Int. J. Eng. Sci., 7, pp. 947-961 (1969).

51. Yang, F., Chong, A.C.M., Lam, D.C.C. and Tong, P. "Couple stress based strain gradient theory for elasticity", Int. J. Solids Struct., 39, pp. 2731-2743 (2002).

52. Rokni, H., Seethaler, R.J., Milani, A.S., Hashemi, S.H. and Li, X.F. "Analytical closed-form solutions for size-dependent static pull-in behavior in electrostatic micro-actuators via Fredholm integral equation", Sens. Actuators, A., 190, pp. 32-43 (2013).

53. Zhang, J. and Fu, Y. "Pull-in analysis of electrically actuated viscoelastic microbeams based on a modified couple stress theory", Meccanica., 47, pp. 1649-1658 (2012).

54. Mindlin, R.D. and Tiersten, H.F. "Effects of couplestresses in linear elasticity", Arch. Ration. Mech. Anal., 11, pp. 415-448 (1962).

55. Dequesnes, M., Rotkin, S.V. and Aluru, N.R. "Calculation of pull-in voltage for carbon-nanotube-based nanoelectromechanical switches", Nanotechnology, 13, pp. 120-131 (2002).

56. Yin, L., Qian, Q. and Wang, L. "Size effect on the static behavior of electrostatically actuated microbeams", Acta Mech. Sin., 27, pp. 445-451 (2011).
57. Rezazadeh, Gh., Vahdat, A.S., Tayefeh-rezaei, S. and Cetinkaya, C. "Thermoelastic damping in a microbeam resonator using modified couple stress theory", Acta Mechanica., 223(6), pp. 1137-1152 (2012).

58. Wang, B., Zhou, S., Zhao, J. and Chen, X. "Sizedependent pull-in instability of electrostatically actuated microbeam-based MEMS", J. Micromech. Microeng., 21, 027001 (2011).

59. Wang, B., Zhou, S., Zhao, J. and Chen, X. "Pullin instability analysis of electrostatically actuated microplate with rectangular shape", Int. J. Precis. Eng. Manuf., 12, pp. 1085-1094 (2011).

60. Wang, B., Zhou, S., Zhao, J. and Chen, X. "Pullin instability of circular plate mems: a new model based on strain gradient elasticity theory", Int. J. Appl. Math., 4, 1250003 (2012).

61. Ansari, R., Gholami, R., Mohammadi, V. and Faghih Shojaei, M. "Size-dependent pull-in instability of hydrostatically and electrostatically actuated circular microplates", Compos. Struct., 95, pp. 430-442 (2013).

62. Mohammadi, V., Ansari, R., Faghih Shojaei, M., Gholami, R. and Sahmani, S. "Size-dependent dynamic pull-in instability of hydrostatically and electrostatically actuated circular microplates", Nonlinear Dyn., 73, pp. 1515-1526 (2013).

63. Rahaeifard, M., Kahrobaiyan, M.H., Ahmadian, M.T. and Firoozbakhsh, K. "Size-dependent pull-in phenomena in nonlinear microbridges", International Journal of Mechanical Sciences, 54(1), pp. 306-310 (2012).

64. Klimchitskaya, G.L., Mohideen, U. and Mostepanenko, V.M. "Casimir and van der Waals forces between two plates or a sphere (lens) above a plate made of real metals", Physical Review A, 61(6), 062107 (2000).

65. Boström, M. and Sernelius, B.E. "Fractional van der Waals interaction between thin metallic films", Physical Review B, 61(3), p. 2204 (2000).

66. Israelachvili, J.N. and Tabor, D. "The measurement of Van Der Waals dispersion forces in the range 1.5 to 130 nm", Proceedings of the Royal Society of London. Series A, Mathematical and Physical Sciences, 331, pp. 19-38 (1972).

67. Teo, L.P. "Casimir piston of real materials and its application to multilayer models", Physical Review A, 81, 032502 (2010).

68. Lin, W-H. and Zhao, Y-P. "Casimir effect on the pullin parameters of nanometer switches", Microsystem Technologies, 11(2-3), pp. 80-85 (2005).

\section{Biographies}

Abolfazl Kanani received the BS degree in Physics from Yazd University and his MSc degree from Faculty of Advanced Sciences and Technologies, University of Isfahan. His current research includes modeling the behavior of nanostructures and nano-materials. 
Ali Koochi received the MSc degrees in Aerospace Engineering from Sharif University of Technology, Tehran, Iran. He is interested in modeling nano-structures and NEMS using nano-scale continuum models.

Mohammad Farahani obtained his $\mathrm{PhD}$ degree in propulsion division from the Sharif University of Technology, Iran, where he is an Assistant Professor in the Aerospace Engineering Department. His research interests include applied aerodynamics, unsteady aerodynamics, wind tunnel testing, gas dynamics, and turbo machinery.

Ehsan Roohi obtained a PhD degree in Aerospace En- gineering from Sharif University of Technology, Tehran, Iran, in 2010, and is currently Assistant Professor of Mechanical Engineering at Ferdowsi University of Mashhad, Iran. His research interests include: rarefied gas dynamics, Direct Simulation Monte Carlo (DSMC) and two phase flows.

Mohamadreza Abadyan received the MSc and PhD degrees in Aerospace Engineering from Sharif University of Technology, Iran, in 2004 and 2010, respectively. $\mathrm{He}$ is currently collaborating with the Islamic Azad University, Shahrekord Branch, Shahrekord, Iran, as a researcher. His current research interests include the pull-in performance of MEMS/NEMS. 